\title{
Retinal thickness changes after phacoemulsification
}

This article was published in the following Dove Press journal:

Clinical Ophthalmology

4 November 2013

Number of times this article has been viewed

\author{
Gede Pardianto' \\ Nila Moeloek ${ }^{2}$ \\ Julia Reveny' \\ Sutarman Wage' \\ Imsyah Satari ${ }^{3}$ \\ Rosita Sembiring' \\ Nuttamon Srisamran ${ }^{4,5}$ \\ 'Sumatera Utara University, Medan, \\ ${ }^{2}$ University of Indonesia, Jakarta, \\ ${ }^{3}$ Sumatera Eye Center, Medan, \\ Indonesia; ${ }^{4}$ Thammasat University, \\ Pathum Thani, Thailand; ${ }^{5}$ The World \\ Medical Center, Nonthaburi, Thailand
}

Purpose: To determine the effect of phacoemulsification on macular volume and thickness using spectral domain optical coherence tomography examinations.

Methods: Twenty-seven eyes of 27 subjects who underwent phacoemulsification were studied. All nine areas of the macula were examined by spectral domain optical coherence tomography preoperatively and 2 months postoperatively. Effective phacoemulsification time and absolute phacoemulsification time were also recorded.

Results: There were statistically significant differences in macular thickness between preoperative and postoperative spectral domain optical coherence tomography examinations in nine areas including macular volume. In the paracentral macular area, the thickness of three quadrants significantly increased (superior $P=0.015$; temporal $P=0.001$; and nasal $P=0.023$ ). Peripheral macular thickness also increased significantly in the superior $(P=0.05)$ and temporal macular areas $(P<0.001)$. The macular volume increased significantly after phacoemulsification $(P<0.001)$. There were no correlations between absolute/effective phacoemulsification time and macular cellular structures $(P>0.05)$, but a significant correlation $(P=0.011)$ was found between absolute phacoemulsification time and change in macular volume.

Conclusion: Macular thickness changes in the nasal, superior, and temporal quadrants of the paracentral area and the superior and temporal quadrants of the peripheral area, as well as macular volume, may be used as detailed biomarkers to measure the effects of intraocular pressure fluctuations and maneuvers in phacoemulsification intraocular surgeries.

Keywords: phacoemulsification, effective phaco time, absolute phaco time, SD-OCT, macula

\section{Introduction}

Phacoemulsification (phaco) is one of the most widely used cataract surgery techniques nowadays. Various factors involved in phaco can influence the tissue structures of the eyeball. Unlike other maneuvers, ultrasonic energy and fluidics produce mechanical effects that cause an inflammatory reaction, compression, and hypoxia on the tissue. Every step of this maneuver can cause direct effects on tissue and instantaneous pressure fluctuation. Fluidics also has radiating pressure effects which resemble a miniature shock wave and jet stream that point directly onto the anterior chamber tissue and are forwarded in all directions. Ultrasonic energy should also be taken into account as a risk factor that may affect the structure of the tissue in the eyeball. In this case, phaco surgeons can measure the ultrasonic energy that was emitted through the phaco time. Phaco time consists of two components: effective phaco time (EPT) and absolute phaco time (APT). Both EPT and APT are representatives of any countable
Correspondence: Gede Pardianto Sumatera Utara University, Iskandar Muda 278, Medan 201 I2, Indonesia

Tel +628155000300

Fax +62614142217

Email gedepardianto@yahoo.com 
ultrasonic energy used during phaco. EPT is a parameter that is calculated by multiplying the total APT by the average percentage of power used, and represents how long phaco time would have been at $100 \%$ phaco power. ${ }^{1-4}$

The retina is a sensory array that requires more oxygen than the brain; the structure is highly sensitive to changes in oxygen levels and changes in the condition of the eyeball. Micro changes in the retina appear as normal on visual inspection using visual ophthalmoscopy. Micro changes are often not felt by the patient, even after severe damage; only later does the patient begin to notice a decline in visual function..$^{5,6}$ The macula is an important structure in charge of the 30-degree field of view, which greatly determines the quality of sharp vision, communication, interpersonal relationships, and color vision, including contrast sensitivity. The central macula has been widely studied, and is closely related to the function of the fovea in visual acuity and color vision. The retinal nerve fiber layer (RNFL) has been of great interest in relation to the peripheral visual field, whereas the paracentral and peripheral macula have been paid much less attention. In everyday life, there is a high correlation between paracentral sight and interpersonal responses, such as courteous responses or attention during communication, and with routine activities from the moment we wake till the time we sleep. The important role of the paracentral and peripheral areas of the macula is needed throughout life. ${ }^{6-11}$

As with other types of eye surgery, phaco is a short process but still has dynamics that contribute to its risk factor. ${ }^{1,3,4,12-14}$ Some scientific research in the field of phaco safety on the central fovea and RNFL have been done, but a comprehensive study of the entire macular area and macular volume has not yet surfaced. Following many studies, a few reports have been published, but these had limitations and used time domain optical coherence tomography (OCT) only. ${ }^{12-17}$

The purpose of this study was to determine the effect of phaco on macular volume and thickness using spectral domain OCT (SD-OCT) examinations.

\section{Materials and methods}

This study performed a quasi-experimental pre-post study based on SD-OCT to estimate the causal impact of phaco and monitor changes in living macular tissue structures its thickness and volume in numerical data. The study was conducted at the Sumatera Eye Center from September 2011 to February 2013. The participants were chosen by simple random sampling. Each participant underwent a comprehensive ophthalmologic examination, including optical biometry and SD-OCT. To be included in the study, participants had to meet the following criteria at their initial assessment: best corrected visual acuity of less than 20/40, presence of a normal anterior chamber, an open angle on slit-lamp and gonioscopic examinations, suitable lens opacities for optical biometry and SD-OCT examinations, intraocular pressure (IOP) $<21 \mathrm{mmHg}$, and willingness to participate in the study. Patients with normal-tension glaucoma, age-related macular degeneration, diabetes mellitus, hypertension, and other systemic diseases were excluded from this study. Optical biometry data were obtained using an optical biometer (IOLMaster ${ }^{\circledR}$ 500; Carl Zeiss Meditec AG, Jena, Germany).

The institutional review board approved this study, and the study design followed the principles of the Declaration of Helsinki. The subjects underwent phaco using the phaco chop technique with bimanual irrigation-aspiration and intraocular lens (IOL) hydroimplantation; a Venturi-based phaco machine was used (Stellaris; Bausch and Lomb Incorporated, Rochester, NY, USA) under an operating microscope (OPMI Lumera $^{\circledR}$; Carl Zeiss Meditec). Hydroimplantation was performed by inserting the IOL without viscoelastic material, and using an irrigation tube to fill the anterior chamber. Phaco was performed by only one surgeon (IS). EPT and APT were recorded.

Before phaco, the eyes were instilled with tropicamide $1 \%$ eye drops (Mydriatil; PT Cendo Pharmaceutical, Indonesia), and diclofenac sodium $0.1 \%$ eye drops (Voltaren ${ }^{\circledR}$ Ophthalmic; Novartis AG, Basel, Switzerland). BSS ${ }^{\circledR}$ sterile irrigating solution (Alcon Laboratories, Inc., Fort Worth, TX, USA), a viscoelastic material $1: 3$ mixture of $4 \%$ chondroitin sulfate, and 3\% sodium hyaluronate (Viscoat ${ }^{\circledR}$; Alcon) were used during phaco. After phaco, the eyes were instilled with moxifloxacin $0.5 \%$ eye drops (Vigamox ${ }^{\circledR}$; Alcon) four times daily and diclofenac sodium $0.1 \%$ eye drops four times daily for 1 week.

SD-OCT images were obtained using a Cirrus ${ }^{\text {TM }}$ HD-OCT 4000 system (Carl Zeiss Meditec). Macular thickness and volume were derived using the macular cube mode. Macular cube data were achieved from a three-dimensional dataset composed of $512 \mathrm{~A}$-scans derived from $128 \mathrm{~B}$-scans that covered a $6 \times 6 \mathrm{~mm}$ area centered on the fovea. Pupil dilatation was performed in all eyes. All accepted images exhibited a centered optic disc or fovea, were well focused, exhibited no eye motion within the measurement circle, and had a signal strength of $\geq 7$. $^{18}$

All subjects were examined in all nine areas of the macula and macular volume by SD-OCT preoperatively 
and 2 months postoperatively. The macula was divided into three areas: foveal (central), inner (paracentral), and outer (peripheral). Inner and outer areas were subdivided into four quadrants: superior, temporal, inferior, and nasal. Additionally, this study used modified names according to the nine macular map sectors of the Early Treatment Diabetic Retinopathy Study (ETDRS): central macula for fovea, paracentral macula for parafovea, and peripheral macula for perifovea. ${ }^{18}$ Macular thickness and volume before and after phaco were recorded.

Baseline characteristics and research data were recorded in a prepared clinical record form. Existing data were compiled, clarified, confirmed, and then tabulated in the specific tables in accordance with the purposes of the study using Microsoft Excel ${ }^{\circledR} 2007$ software (Microsoft Corporation, Redmond, WA, USA). The results of macular thickness and volume measurements in numerical data included the macular area shortly before the surgery and 2 months postoperatively.

Statistical analyses were performed using IBM SPSS ${ }^{\circledR}$ Statistics version 19.0 (IBM Corporation, Armonk, NY, USA). Descriptive statistics are presented as mean \pm standard deviation. The differences of preoperative and postoperative data sets were identified by paired Student's $t$-test. $P<0.05$ was considered the level of statistical significance. Correlations between phaco time and structural changes in the macula were analyzed by Pearson's correlation test. The size of the correlation coefficient $(r)$ was categorized according to the method proposed by Borenstein and Cohen: 0.0-0.09, no correlation; $0.1-0.3$, small correlation; $0.3-0.5$, medium correlation; and $0.5-1.0$, strong correlation. ${ }^{19}$

\section{Results}

A total of 27 eyes of 27 immature cataract patients were included in this study. Baseline clinical characteristics of participants are presented in Table 1. The mean age was $64.83 \pm 15.45$ years (43-79 years). Female patients (18 eyes, $67 \%$ ) were predominant. There were slightly more right eyes (16 eyes, 59\%) than left eyes. All participants were Indonesian and none of them had previous cataract surgery.

The preoperative and postoperative thicknesses of the central, paracentral, and peripheral macular areas are shown in Table 2. An example of an OCT image before and after phaco is illustrated in Figure 1. There was an insignificant increase in thickness in the central macular area $(214.07 \pm 59.95 \mu \mathrm{m}$ versus $236.67 \pm 63.67 \mu \mathrm{m}$, respectively; $P=0.068)$. The thickness of all quadrants, except the inferior sector $(275.00 \pm 62.87 \mu \mathrm{m}$ versus $288.33 \pm 63.00 \mu \mathrm{m}$;
Table I Baseline characteristics

\begin{tabular}{ll}
\hline Characteristics & $\begin{array}{l}\text { Patients or } \\
\text { eyes }\end{array}$ \\
\hline $\begin{array}{l}\text { Age at baseline, years } \\
\text { Sex, number of eyes (\%) } \\
\text { Male }\end{array}$ & $64.83 \pm 15.45$ \\
Female & $18(67)$ \\
Race, number of eyes (\%) & $9(33)$ \\
Indonesian Malay & \\
Indonesian Chinese & $18(59)$ \\
Eye laterality, number of eyes (\%) & $9(4 I)$ \\
Right & \\
Left & $16(59)$ \\
Previous cataract surgery, number of eyes (\%) & $11(4 I)$ \\
Yes & $0(0)$ \\
No & $27(100)$ \\
\hline
\end{tabular}

$P=0.388)$, in the paracentral area significantly increased (superior: 269.93 $\pm 64.13 \mu \mathrm{m}$ versus 303.85 $\pm 40.81 \mu \mathrm{m}$; temporal: $238.26 \pm 83.95 \mu \mathrm{m}$ versus $291.11 \pm 42.12 \mu \mathrm{m}$; and nasal: $277.96 \pm 53.61 \mu \mathrm{m}$ versus $303.96 \pm 32.87 \mu \mathrm{m} ; P=0.015$, 0.001 , and 0.023 , respectively). Peripheral macular thickness also increased significantly in the superior (227.19 $\pm 69.81 \mu \mathrm{m}$ versus $269.48 \pm 25.60 \mu \mathrm{m} ; P=0.005$ ) and temporal (180.81 $\pm 97.37 \mu \mathrm{m}$ versus $252.15 \pm 31.80 \mu \mathrm{m}$; $P<0.001)$ quadrants. Similar to macular thickness in most areas, the macular volume also significantly increased after phaco $\left(7.85 \pm 1.78 \mu \mathrm{m}^{3}\right.$ versus $\left.9.46 \pm 1.05 \mu \mathrm{m}^{3} ; P<0.001\right)$. The maximal and minimal differences of mean macular thickness were revealed in the peripheral temporal and paracentral inferior sectors (71.33 $\mu \mathrm{m}$ versus $13.33 \mu \mathrm{m}$, respectively).

EPT and APT were 14.30 111.43 seconds and $24.52 \pm 19.87$ seconds, respectively. In global analyses of

Table 2 Preoperative and postoperative macular thickness and volume after phacoemulsification

\begin{tabular}{|c|c|c|c|c|}
\hline & \multicolumn{2}{|c|}{ Phacoemulsification } & \multirow{2}{*}{$\begin{array}{l}\text { Mean } \\
\text { difference }\end{array}$} & \multirow[t]{2}{*}{$P$-value } \\
\hline & Preoperative & Postoperative & & \\
\hline \multicolumn{5}{|c|}{ Macular thickness $(\mu \mathrm{m})$} \\
\hline Central & $214.07 \pm 59.95$ & $236.67 \pm 63.67$ & 22.60 & 0.068 \\
\hline \multicolumn{5}{|c|}{ Paracentral } \\
\hline Superior & $269.93 \pm 64.13$ & $303.85 \pm 40.81$ & 33.93 & 0.015 \\
\hline Temporal & $238.26 \pm 83.95$ & $291.11 \pm 42.12$ & 52.85 & 0.001 \\
\hline Inferior & $275.00 \pm 62.87$ & $288.33 \pm 63.00$ & 13.33 & 0.388 \\
\hline Nasal & $277.96 \pm 53.61$ & $303.96 \pm 32.87$ & 15.96 & 0.023 \\
\hline \multicolumn{5}{|c|}{ Peripheral } \\
\hline Superior & $227.19 \pm 69.81$ & $269.48 \pm 25.60$ & 42.30 & 0.005 \\
\hline Temporal & $|80.8| \pm 97.37$ & $252.15 \pm 31.80$ & 71.33 & $<0.001$ \\
\hline Inferior & $247.63 \pm 56.45$ & $262.4 I \pm 28.75$ & 14.78 & 0.090 \\
\hline Nasal & $272.93 \pm 53.62$ & $288.89 \pm 25.77$ & 15.96 & 0.130 \\
\hline $\begin{array}{l}\text { Volume } \\
\left(\mu \mathrm{m}^{3}\right)\end{array}$ & $7.85 \pm 1.78$ & $9.46 \pm 1.05$ & 1.60 & $<0.001$ \\
\hline
\end{tabular}



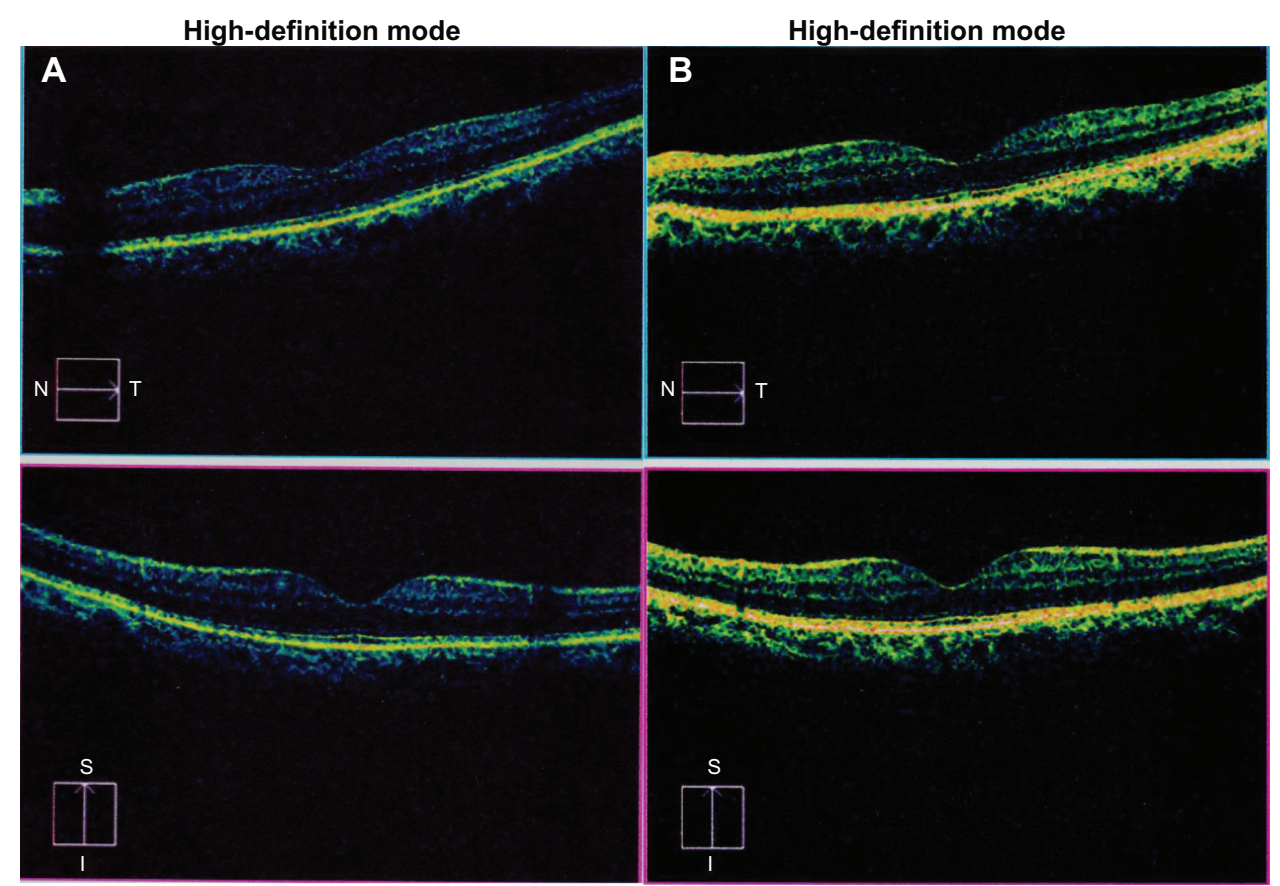

Figure I Spectral domain optical coherence tomography image of the left eye (A) before and (B) after phacoemulsification. Note: N-T is from nasal to temporal; I-S is from inferior to superior.

this study, there were no significant correlations between EPT/APT and the overall changes in macular thickness (Table 3). The maximum and minimum correlation of EPT and macular thickness change was found in the paracentral temporal and peripheral temporal sectors $(0.421$ versus 0.016 , respectively). No strong correlations were found in any sector. A medium correlation was found in the paracentral temporal sector only $(r=0.421)$.

The maximum and minimum correlation of APT and macular thickness change was found in the peripheral

Table 3 Correlation of phacoemulsification time and structural changes in the macula

\begin{tabular}{|c|c|c|}
\hline & $\begin{array}{l}\text { Effective } \\
\text { phacoemulsification } \\
\text { time }\end{array}$ & $\begin{array}{l}\text { Absolute } \\
\text { phacoemulsification } \\
\text { time }\end{array}$ \\
\hline & \multicolumn{2}{|c|}{ Correlation coefficient ( $P$-value) } \\
\hline \multicolumn{3}{|c|}{ Macular thickness $(\mu \mathrm{m})$} \\
\hline Central & $0.290(0.143)$ & $0.168(0.403)$ \\
\hline \multicolumn{3}{|l|}{ Paracentral } \\
\hline Superior & $0.161(0.421)$ & $0.109(0.588)$ \\
\hline Temporal & $0.421(0.703)$ & $0.035(0.861)$ \\
\hline Inferior & $0.111(0.580)$ & $0.06 I(0.76 I)$ \\
\hline Nasal & $0.175(0.383)$ & $0.075(0.7 \mathrm{II})$ \\
\hline \multicolumn{3}{|l|}{ Peripheral } \\
\hline Superior & $0.185(0.356)$ & $0.177(0.377)$ \\
\hline Temporal & $0.016(0.938)$ & $0.326(0.097)$ \\
\hline Inferior & $0.076(0.706)$ & $0.175(0.384)$ \\
\hline Nasal & $0.175(0.383)$ & $0.075(0.7 \mathrm{II})$ \\
\hline Volume $\left(\mu \mathrm{m}^{3}\right)$ & $0.167(0.405)$ & $0.487(0.011)$ \\
\hline
\end{tabular}

temporal and paracentral temporal sectors $(0.326$ versus 0.035 , respectively). No strong correlations were found. A medium correlation was found in the peripheral temporal sector only ( $r=0.326)$. In addition, EPT showed a small correlation with macular volume change, but this was not significant ( $r=0.167 ; P=0.405)$; APT showed a significant medium correlation $(r=0.487 ; P=0.011)$.

The visual acuity and IOP before and after phaco are shown in Table 4. Postoperative uncorrected and best corrected visual acuity significantly improved $(P<0.001$ and $P<0.001$, respectively). On the other hand, postoperative IOP significantly decreased $(16.96 \pm 2.85 \mathrm{mmHg}$ versus $15.30 \pm 2.51 \mathrm{mmHg}, P<0.001)$.

\section{Discussion}

Phaco is a current technique that uses ultrasonic energy to remove the cataract lens, which is then replaced with an IOL. Various studies have shown that phaco gives satisfactory results and is safe for patients, makes a major contribution in the treatment, and avoids any complications of cataract blindness, as well as being scientifically proven safe enough for the cornea, anterior chamber angle, and iris. Foveal thickness changes associated with phaco have also been reported. Studies on the common effects of phaco on the corneal endothelium, RNFL, and macula are numerous but comprehensive research concerning the particular effects of ultrasonic energy delivered by phaco on the macula has not been performed. ${ }^{12-15,18}$ 
Table 4 Preoperative and postoperative visual acuity and intraocular pressure after phacoemulsification

\begin{tabular}{clll}
\hline & \multicolumn{2}{l}{ Phacoemulsification } & P-value \\
\cline { 2 - 3 } & $\begin{array}{l}\text { Preoperative } \\
\text { (mean) }\end{array}$ & $\begin{array}{l}\text { Postoperative } \\
\text { (mean) }\end{array}$ & \\
\hline Visual acuity (decimal) & & & \\
Uncorrected & $0.17 \pm 0.177$ & $0.67 \pm 0.294$ & $<0.00$ I \\
& $(0.025-0.66)$ & $(0.1-1.0)$ & \\
Best corrected & $0.23 \pm 0.134$ & $0.94 \pm 0.134$ & $<0.00 \mathrm{I}$ \\
& $(0.05-0.66)$ & $(0.66-1.0)$ & \\
Intraocular pressure & $16.96 \pm 2.85$ & $15.30 \pm 2.5 \mathrm{I}$ & $<0.00 \mathrm{I}$ \\
(mmHg) & $(12-20)$ & $(\mathrm{II}-20)$ & \\
\hline
\end{tabular}

Note: Data expressed as mean \pm standard deviation (range).

Briefly, IOP fluctuation occurring in eye surgery is also suspected to cause damage to the RNFL. On the contrary, phaco has also been found to increase the thickness of the retina and cystoid macular edema. ${ }^{12}$ In the present study, no significant change in the central macular area was found before and after phaco, which differs from many previous studies. $^{12-14}$

OCT is a diagnostic tool which is noncontact, noninvasive, and capable of displaying slices of living tissue with high resolution (high definition). It operates on the principle of coherence interferometry using infrared light with high reliability and high validity, and does not require immersion. OCT has evolved through several generations from the prototype of time domain OCT to the latest generation of Fourier domain OCT or SD-OCT. ${ }^{18}$

SD-OCT uses the principle of spectral detectors for infrared light with a wavelength of about $840 \mu \mathrm{m}$. Its display performance can exceed 28,000 A-scans per second with an axial resolution of $1-5 \mu \mathrm{m}$ and a transverse resolution of $5-10 \mu \mathrm{m}$. The maximum A-scans per B-scan is 8,000 to a depth of $2 \mathrm{~mm}$ scan, thus presenting a higher resolution, examination 50-100 times faster, and providing more information than its predecessor. ${ }^{18}$

SD-OCT is a diagnostic tool for the tissue layer of the macula and retina with $>87 \%$ sensitivity and $>98 \%$ specificity. Macular examination displayed in nine sectors according to the Early Treatment Diabetic Retinopathy Study (ETDRS). ${ }^{18}$ SD-OCT can help explain the shape and histology of the optic nerve head, retina, and macula with excellent accuracy, validity, and reproducibility with very high reliability. SD-OCT is not complicated to operate and the results are easily interpreted due to the derived clarity of each layer of the retina and macula, which can help discriminate the pathology from normality. In addition, an OCT macular scan is based on an eye tracker feature that can scan the same location with high accuracy on serial examinations. $^{18}$

On examination of the paracentral macula in the present study, significant increases in thickness were found in nearly all sectors, except the inferior part. This significant change was also found in the superior and temporal sectors of the peripheral macula. The present study did not find any pathological disorders in postoperative macular thickening, and the increased thicknesses were still within normal range in accordance with the normative database contained in the SD-OCT software. On obtained OCT images, pathologic abnormalities such as tissue edema, anomaly on each layer, detachment of one layer from another layer, accumulation of fluid between layers, and deformation or damage that could be detected by SD-OCT were not found. Mild foveal and perifoveal edema after phaco was not demonstrated by SD-OCT and was only demonstrated by time domain OCT. In addition, this mild edema with nonspecific area can occur without visual dysfunction. One possible explanation of the cause of postoperative macular edema is damage to the blood-retinal barrier, which could induce consequent macular thickening. ${ }^{20,21}$

The concordance of thickness change in the superior and temporal sectors of both the paracentral and peripheral macula may be a new indicator to help explain the susceptibility patterns of the retina associated with various factors in phaco, eg, length of surgery, phaco time, phaco energy. In other intraocular surgeries or any harmful situations that can cause damage to the RNFL, these features may also occur and indirectly represent the impact of invasive manipulations of the eyeball, which potentially cause some degree of change in the tissue structure of the retina.

According to normal anatomy, the RNFL is a direct continuation of the retinal ganglion cell layer, and is mostly centripetal fibers. The distribution of fibers around the nerve head is nonuniform and thicker at the superior and inferior poles. However, the superior part is found to be slightly thinner than the inferior part. In addition, the macular fibers are aligned horizontally, form the papillomacular bundle, and enter the optic nerve on the temporal side. The temporal fibers of the optic disc that arise more peripheral to the papillomacular bundle have to arch over the bundle to reach the optic nerve as so-called arcuate fibers, whereas the fibers from the nasal side of the optic disc are located in a more radial fashion. ${ }^{5,6,8,9}$ Peripheral fibers occupy more peripheral locations in the nerve head and more superficial positions closer to the vitreous, compatible with the fact that the temporal area has long been known as the weakest part of the 
retina and could be an early sign of RNFL abnormalities and disorders of the visual field..$^{5-9}$

In the present study, there was an insignificant increase in the central macular area $(P=0.068)$. However, this value must not be regarded simply and confidently as insignificant. A larger study sample may probably give a different conclusion. Furthermore, it was interesting to see that the pattern of change in RNFL thickness peripheral to the macula after phaco was similar to that of RNFL loss in glaucoma. Concerning the mean differences of macular thickness before and after phaco, the temporal sector had greater change than the superior sector in both the paracentral and peripheral macular regions. It could be assumed that the change originated from the temporal area of the macula and then continued toward the superior area. In addition, it occurred in both the paracentral and peripheral macular regions in parallel fashion, comparable to RNFL thinning in glaucoma which also starts in the temporal area. Interestingly, RNFL change in the present study occurred earlier in the superior part. This additional finding supported the aforementioned fact that the superior RNFL is thinner, ie, more vulnerable than the inferior part. This interpretation is based only on histologically topographic changes; functional changes in the retina also need to be evaluated.

On the other hand, the macular volume also revealed statistically significant changes. In the analysis of each retinal layer, there were no pathological abnormalities or tissue edema found. Still, increased thickening of the macula was classified as normal thickness following the normative database contained in the SD-OCT software. This study also indicates that the macular volume was determined not only by the thickness of the central area of the macula or fovea but also by the thickness of the paracentral and peripheral areas. Changes in macular volume may be a new biomarker for retinal structure changes in intraocular surgeries. Even though the statistically significant change was still in the normal category compared to the normative database, it could be a reference for more in-depth study on this issue. However, the interference of the blood-retinal barrier due to intraocular surgery should always be considered.

Clear media possibly gives better OCT penetration, and the changes should be found in all areas. However, the present study found that it was not in all areas, so the authors believe that there are other factors that cause changes in specific areas of the macula beyond the cleared media. The increases in thickness, although statistically significant, never exceeded normal limits. Some specific changes in thick- ness distinguishes this study's results from similar reports in the literature. These results are quite interesting and represent a valuable contribution to improving maneuvers during phaco.

In this study, there were no significant correlations between EPT and APT with the changes in tissue structure of the central, paracentral, and peripheral macular areas. Interestingly, macular volume did not have a significant correlation with EPT, but did with APT.

The absence of a significant correlation suggests two possible causes. First, ultrasonic energy might not affect macular tissue structures. Second, the changes occurred because of maneuverability of the surgeons, detailed intraoperative IOP fluctuations, and inflammation. IOP fluctuations may occur due to the dynamics of fluidics (irrigation fluid with wave pressure and specific power, spinning, and flowing on certain tissues in the eyeball) at each step of the operation. This condition can also occur in any intraocular surgery procedure, including cataract and refractive surgeries.

IOP and foveal thickness after phaco were also studied. RNFL thickness change was reported after photorefractive surgery that used no ultrasound power, but IOP fluctuation existed due to a high vacuum. Increased IOP associated with the microkeratome vacuum ring used during laser in situ keratomileusis may have precipitated optic nerve head ischemia and visual field defects. ${ }^{22}$ RNFL thickness was found to be significantly thinner after a single attack of primary acute angle-closure glaucoma due to short significant IOP fluctuation. ${ }^{23}$

In the present study, postoperative IOP data decreased at 1 month and 2 months and seemed stable. On the other hand, there was an initial increase in foveal thickness, followed by a significant decrease at 9 hours and 12 hours, and then an increase in thickness again at 1 month and 2 months follow-up. Interestingly, the increase in eye pressure and foveal thickness showed a significantly negative correlation.

Practically, IOP fluctuation during phaco in intact eyes is still difficult to estimate due to the rare opportunities to measure, efficient instruments, position and constant location of the instrument, increased risk of infection from frequent measurements, and ethical problems. A limitation of the present study is the measurement of IOP fluctuation during the surgery, which was difficult to observe due to patient safety and patient comfort regulations.

From the present results, both EPT and APT showed no relationship with macular thickness. This may suggest that the delivered ultrasonic energy onto the retinal structures was low and phaco was safe for retinal structures. To help 
decrease intraoperative IOP fluctuation, various techniques can be applied, eg, proper ophthalmic viscoelastic devices' use, gradual filling of the appropriate volume of ophthalmic viscoelastic devices into the anterior chamber, gentle hydrodissection, consistently maintaining the volume of the anterior chamber during surgery, bottle height adjustment, competent maneuverability, concise operation. Nonetheless, controlling postoperative inflammation is also noteworthy.

In the present study, the medium correlation between APT and macular volume implies that the macular volume was susceptible to the exposure of ultrasonic energy. Even though ultrasonic energy is generally safe to macular tissue structures, excessive exposure should still be avoided. For this reason, the surgeon is advised to stay focused on the EPT, not hesitate to segment removal, and not push down the pedal too long to reduce the APT and minimize the ultrasonic energy delivery and its effects on the macular volume.

The strength of this study is revealing that macular thickness changes in the superior and temporal sectors of the paracentral and peripheral macular areas, as well as macular volume, may be used as more detailed biomarkers to measure the effects of IOP fluctuations and skillful proceedings in phaco. From this study, there were no correlations between APT/EPT and macular thickness changes in phaco, but there is correlation between APT and macular volume change. Macular volume was determined not only by the thickness of the central area of the macula or fovea, but also influenced by the thickness of the paracentral and peripheral areas. Thus, macular volume may be useful in observing the effect of APT on tissue structures of the retina.

This study contributes advice and supports the theories about the importance of RNFL and macular assessment by OCT before cataract surgery. OCT can be considered for macular evaluation before cataract surgery to confirm the status of visual acuity and RNFL thickness as a predictor of outcome after cataract surgery in immature cases, especially in the era of multifocal and premium IOLs. OCT follow-up should be routinely performed in high-risk patients whose RNFL has a tendency to be damaged, to help guarantee sight after surgery. The necessity of macular examination by SD-OCT prior to phaco is highly recommended and mandatory especially in premium IOL implantation to ensure the absence of minimal retinal abnormalities that appear normal in other ophthalmoscopic exams. Moreover, SD-OCT follow-up examination is also recommended in patients who have prolonged phaco, preexisting high IOP and/ or high IOP fluctuation during surgery, a lot of irrigation fluid consumption, intraoperative complications, shallow anterior chamber, hard and/or large cataract, and patients with possible retinal disorders such as diabetic retinopathy and macular degeneration.

Both uncorrected and best corrected visual acuities after phaco improved significantly following cataract extraction and IOL implantation. Visual gain was higher with best correction. The results of the numerical analyses corroborated the change in tissue structures of the retina that contribute to the successful outcome of surgery. In addition, these results supported the safety of performing phaco on retinal tissue structures.

The results showed a statistically significant difference between preoperative and postoperative IOP. Many factors should be taken into account. Phaco helped remove the thick lens which blocked the aqueous outflow inside the eyeball, opened the angle of the anterior chamber, improved aqueous outflow, and ultimately lowered the IOP. This study supports phaco as one procedure of choice for glaucoma treatment and should be done in any retinal disorders which have a greater potential of damage and can bring further difficulties if the procedure is delayed. Thus, early phaco can be an indication and applied to the eyes with the following conditions: high IOP, shallow anterior chamber, narrow angle, and thicker lens.

IOP fluctuation after phaco is one predictor of phaco safety to ensure patients gain stable visual function for a long time. To preserve the RNFL and macula, a significant IOP decrease makes phaco become safer both intraoperatively and postoperatively. These results reinforce the fact that phaco is safe for retinal cell structures and ensure the long-term stability of visual function. To ensure the longterm stability of visual function, some modified techniques and improvement in skill and maneuvers during surgery may be necessary to help reduce IOP fluctuations. Macular thickness measurements at longer postoperative intervals may be important.

This study is different from others in detailed sectors and specific areas of the macula. Some sectors may be used as detailed biomarkers in any manipulation of intraocular surgery. Specific sectors changed, but never beyond the normal limit. Therefore, although phaco statistically impacts the macula, it is still clinically safe.

Of note, a detailed SD-OCT scan is highly recommended prior to phaco and should be mandatory in premium IOL implantation to ensure the absence of minimal retinal abnormalities that appear visually normal in routine ophthalmologic exams. 


\section{Acknowledgments}

The authors thank Prof Dr Sjamsu Boediono, Dr Andika Prahasta, Dr Anang Tribowo, Dr Apsari D Kusumastuti, and Andrew P Watts for their support and advice.

\section{Disclosure}

The authors report no conflicts of interest in this work.

\section{References}

1. Park JH, Lee SM, Kwon JW, et al. Ultrasound energy in phacoemulsification: a comparative analysis of phaco-chop and stop-and-chop techniques according to the degree of nuclear density. Ophthalmic Surg Lasers Imaging. 2010;41(2):236-241.

2. Linebarger EJ, Hardten DR, Shah GK, Lindstrom RL. Phacoemulsification and modern cataract surgery. Surv Ophthalmol. 1999;44(2):123-147.

3. Pirazzoli G, D'Eliseo D, Ziosi M,Acciarri R. Effects of phacoemulsification time on the corneal endothelium using phacofracture and phaco chop techniques. J Cataract Refract Surg. 1996;22(7):967-969.

4. Fine IH, Packer M, Hoffman RS. Use of power modulations in phacoemulsification. Choo-choo chop and flip phacoemulsification. J Cataract Refract Surg. 2001;27(2):188-197.

5. Jain KK. Textbook of Hyperbaric Medicine. 4th ed. Cambridge, MA: Hogrefe and Huber; 1990.

6. Hee MR, Izatt JA, Swanson EA, et al. Optical coherence tomography of the human retina. Arch Ophthalmol. 1995;113(3):325-332.

7. Puliafito CA, Hee MR, Lin CP, et al. Imaging of macular diseases with optical coherence tomography. Ophthalmology. 1995;102(2):217-229.

8. Cabrera Fernandez D, Salinas HM, Puliafito CA. Automated detection of retinal layer structures on optical coherence tomography images. Opt Express. 2005;13(25):10200-10216.

9. Cabrera DeBuc D, Somfai GM. Early detection of retinal thickness changes in diabetes using optical coherence tomography. Med Sci Monit. 2010;16(3):MT15-MT21.

10. Hee MR, Puliafito CA, Wong C, et al. Quantitative assessment of macular edema with optical coherence tomography. Arch Ophthalmol. 1995;113(8):1019-1029.

11. Cabrera DeBuc D, Somfai GM, Ranganathan S, Tatrai E, Puliafito CA, Ferencz M. Reliability and reproducibility of macular segmentation using a custom-built optical coherence tomography retinal image analysis software. J Biomed Opt. 2009;14(6):064023.

Clinical Ophthalmology

\section{Publish your work in this journal}

Clinical Ophthalmology is an international, peer-reviewed journal covering all subspecialties within ophthalmology. Key topics include: Optometry; Visual science; Pharmacology and drug therapy in eye diseases; Basic Sciences; Primary and Secondary eye care; Patient Safety and Quality of Care Improvements. This journal is indexed on Submit your manuscript here: http://www.dovepress.com/clinical-ophthalmology-journal
12. Mentes J, Erakgun T, Afrashi F, Kerci G. Incidence of cystoid macular edema after uncomplicated phacoemulsification. Ophthalmologica. 2003;217(6):408-412.

13. Biro Z, Balla Z, Kovacs B. Change of foveal and perifoveal thickness measured by OCT after phacoemulsification and IOL implantation. Eye (Lond). 2008;22(1):8-12.

14. Cagini CF, Fiori T, Iaccheri B, Piccinelli F, Ricci MA, Fruttini D. Macular thickness measured by optical coherence tomography in a healthy population before and after uncomplicated cataract phacoemulsification surgery. Curr Eye Res. 2009;34(12):1036-1041.

15. von Jagow B, Ohrloff C, Kohnen T. Macular thickness after uneventful cataract surgery determined by optical coherence tomography. Graefes Arch Clin Exp Opthalmol. 2007;245(12):1765-1771.

16. Lobo CL, Faria PM, Soares MA, Bernardes RC, Cunha-Vaz JG. Macular alterations after small-incision cataract surgery. J Catarct Refract Surg. 2004;30(4):752-760.

17. Neal RE, Bettelheim FA, Lin C, Winn KC, Garland JS, Zigler JS Jr. Alterations in human vitreous humour following cataract extraction. Exp Eye Res. 2005;80(3):337-347.

18. Tatrai E, Ranganathan S, Ferencz M, Cabrera DeBuc D, Somfai GM. Comparison of retinal thickness by Fourier-domain optical coherence tomography and OCT retinal image analysis software segmentation analysis derived from Stratus optical coherence tomography images. J Biomed Opt. 2011;16(5):056004.

19. Borenstein M, Cohen J. Statistical Power Analysis: A Computer Program. Hillsdale, NJ: Lawrence Erlbaum; 1988.

20. Kusbeci T, Eryigit L, Yavas G, Inan UU. Evaluation of cystoid macular edema using optical coherence tomography and fundus fluorescein angiography after uncomplicated phacoemulsification surgery. Curr Eye Res. 2012;37(4):327-333.

21. Vinores SA, Amin A, Derevjanik NL, Green WR, Campochiaro PA. Immunohistochemical localization of blood-retinal barrier breakdown sites associated with post-surgical macular oedema. Histochem J. 1994;26(8):655-665.

22. Bushley DM, Parmley VC, Paglen P. Visual field defect associated with laser in situ keratomileusis. Am J Ophthalmol. 2000;129(5): 668-671.

23. Wong IY, Yuen NS, Chan CW. Retinal nerve fiber layer thickness after a single attack of primary acute angle-closure glaucoma with optical coherence tomography. Ophthalmic Surg Lasers Imaging. 2010;41(1):96-99.
PubMed Central and CAS, and is the official journal of The Society of Clinical Ophthalmology (SCO). The manuscript management system is completely online and includes a very quick and fair peer-review system, which is all easy to use. Visit http://www.dovepress.com/ testimonials.php to read real quotes from published authors. 